\title{
COMMUNICATION
}

\section{Réhabilitation implantaire avec mise en charge prothétique instantanée et reconstruction par lambeau libre de péroné en un temps.}

\author{
Rohart J1, Afota F2, Dassonville 03, Hauschild U4, Savoldelli C5 \\ 1. Assistant Spécialiste en Chirurgie Orale - Service de chirurgie Orale et Maxillo faciale - CHU Rouen \\ 2. Interne en Chirurgie Orale - Service de Chirurgie Orale et Maxillo Faciale - Institut Universitaire de la Face et du Cou - CHU Nice \\ 3. Chef de Département de Cancérologie ORL - Institut Universitaire de la Face et du Cou - CHU Nice \\ 4. Prothésiste Dentaire, Dental Excellence - San Remo, Italie \\ 5. MCU - PH, Service de Chirurgie Orale et Maxillo Faciale - Institut Universitaire de la Face et du Cou - CHU Nice
}

\section{Introduction}

L'essor de la chirurgie virtuelle et des techniques de conception et fabrication assistée par ordinateur (CFAO) a permis la planification d'importante reconstruction maxillo-faciale et la mise au point de guides sur-mesure de coupe fibulaires par prototypage rapide. Au-delà de ces possibilités, la prévisibilité de la conformation obtenue a également autorisé de prévoir d'emblée la réhabilitation dentaire sur implants avec fiabilité. Nous présentons les étapes de la planification et de la technique chirurgicale d'une reconstruction par lambeau de fibula assistée par guides sur-mesure et la mise en place d'implants en simultanée avec mise en charge prothétique instantanée.

\section{Observation}

II s'agit d'un jeune patient présentant un améloblastome récidivant de l'ensemble de la branche horizontale mandibulaire gauche allant jusqu'au niveau parasymphysaire droit. Le projet prothétique est le point de départ de la planification. La première étape consiste en la simulation de la conformation du lambeau microanatomosé de fibula en fonction de la résection osseuse envisagée. La deuxième étape correspond à la superposition numérique du scanner pré-opératoire avec la planification de la reconstruction mandibulaire. La position implantaire via le logiciel Simplant@ est alors simulée pour correspondre le plus fidèlement possible à l'arcade dentaire initial. Cette étape est essentielle car elle permet de placer virtuellement les implants par rapport à la future prothèse qui sera sensiblement identique aux dents du patient. Une fois la simulation implantaire validée, le guide chirurgical implantaire SAFE SurgiGuide®(Dentsply®) à appui osseux est fabriqué par stéréolithographie après validation du projet global par le chirurgien et le prothésiste. Les implants sont positionnés directement au site de prélèvement fibulaire grâce au guide implantaire. Après ostéosynthèse de la reconstruction osseuse, nous réalisons la mise en fonction instantanée des implants avec la prothèse confectionnée à partir des données numériques préalables. Ce mode opératoire est la meilleure garantie du bon positionnement de la reconstruction, en accord avec les impératifs prothétiques. La mise en charge instantanée permet de guider le patient vers son occlusion d'origine et évite les latérodéviations liées à ce type de chirurgie. Elle permet également une maturation des tissus mous autour de la prothèse provisoire et facilite les étapes de prothèse définitives.

\section{Discussion}

La réhabilitation prothétique de ces patients est un challenge car la prothèse conventionnelle atteint ses limites dans un environnement buccal profondément modifié. Le développement de l'implantologie puis de la planification assistée par ordinateur ont permis de proposer aux patients une réhabilitation prothétique plus adaptée sur le plan fonctionnel et morphologique.

justine.rohart04@gmail.com

(C) The authors, published by EDP Sciences. This is an Open Access article distributed under the terms of the Creative Commons Attribution License 4.0 (http://creativecommons.org/licenses/by/4.0/). 